\title{
Characteristics Comparison of Er-Doped Double-Pass Superfluorescent Fiber Sources Pumped Near $980 \mathrm{~nm}$
}

\author{
L. A. Wang and C. D. Chen
}

\begin{abstract}
The Er-doped superfluorescent fiber sources (SFS's) pumped near $980 \mathrm{~nm}$ in double-pass forward and backward configurations are compared for the first time. The results indicate that a double-pass backward SFS is superior to the forward counterpart in many aspects: higher output power at various lengths of erbium-doped fiber, better pump-power dependent mean wavelength stability, and larger linewidths at various fiber mirror reflectance.
\end{abstract}

Index Terms-Superfluorescent fiber source.

\section{INTRODUCTION}

$\mathbf{R}$ ECENTLY diode-pumped Er-doped superfluorescent fiber sources (SFS's) have been extensively studied because they may serve as light sources in the navigationgrade fiber optical gyroscopes (FOG's) [1], [2]. Among many Er-doped SFS configurations pumped near $980 \mathrm{~nm}$, the singlepass backward (SPB) one has been commonly adopted, and shown to have pump-power independent mean wavelength operation, i.e., $\partial \bar{\lambda} / \partial P_{\text {pump }}=0$ [2]-[4]. In contrast, most reports on SFS's are mainly the simulation works, and focus on the double pass forward (DPF) configuration [1], [5]. Although an SFS in DPF configuration could have a pump-power insensitive mean wavelength operation with a reduced pump efficiency [1], there are no experimental results up to date. Conversely, an Er-doped SFS in the double pass backward (DPB) configuration has been shown to have $\partial \bar{\lambda}_{\text {source }} / \partial P_{\text {pump }}=0$ operations with even broader linewidths than those in the SPB configuration [6]. However, to the best of our knowledge, no experimental results have been reported on the comparison of Er-doped SFS's in both DPF and DPB configurations. We have investigated the SFS's in double pass configurations, and report in this letter the results regarding the following aspects: the dependence of output power on EDF length, pump-power dependent mean wavelength stability, and the effects of the mirror reflectance on output power and linewidths.

Manuscript received October 22, 1996; revised December 17, 1996. This work was supported in part by the National Science Council, Taiwan, R.O.C. under Contract CS 84-0210-D-002-008.

L. A. Wang is with the Institute of Electro-Optical Engineering, National Taiwan University, Taipei, Taiwan, R.O.C. He is also with the Department of Electrical Engineering, National Taiwan University, Taipei, Taiwan, R.O.C.

C. D. Chen is with the Institute of Electro-Optical Engineering, National Taiwan University, Taipei, Taiwan, R.O.C.

Publisher Item Identifier S 1041-1135(97)02408-7.



(a)

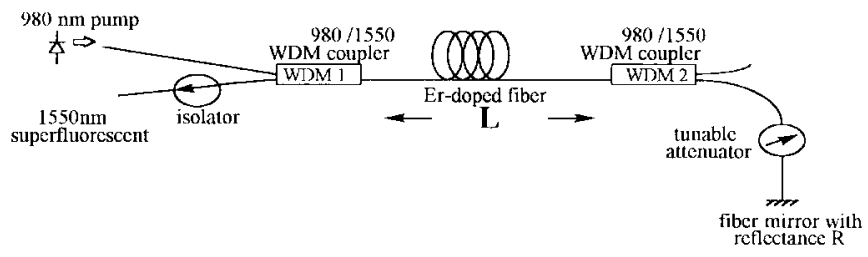

(b)

Fig. 1. Experimental arrangement for the characterization of Er-doped SFS's in (a) DPF and (b) DPB configurations.

\section{EXPERIMENT AND DISCUSSION}

The experimental arrangement used to characterize Erdoped SFS's in double-pass configurations is schematically shown in Fig. 1. Pump power was provided by a laser diode with the maximum output power of $82 \mathrm{~mW}$ measured at the EDF input end, i.e., after WDM1. The EDF with numerical aperture 0.16 was doped with $\sim 600$ wt ppm concentration and codoped with $\mathrm{Al}_{2} \mathrm{O}_{3}$ (1 mole \%) and $\mathrm{GeO}_{2}$ (7 mole \%). The $\mathrm{LP}_{11}$ cutoff wavelength was $\sim 975 \mathrm{~nm}$, and the attentuation was $\sim 10.5 \mathrm{~dB} / \mathrm{m}$ measured at $1530 \mathrm{~nm}$. A fiber mirror with controllable reflectance realized by incorporating a fiber mirror $(R \sim 90 \%)$ and a tunable precision attenuator was spliced to one of the WDM's to reflect the backward and the forward ASE signals in DPF and DPB configurations, respectively. In order to reduce the optical feedback at the signal output end, a polarization-insensitive isolator with $-59-\mathrm{dB}$ isolation was incorporated. The residual pump end of WDM2 was applied with index matched oil to prevent back reflection. The mean wavelength [3], linewidth [1], and pumping efficiency were calculated based on the measured spectra.

Shown in Fig. 2 is the output power measured after the isolator's output port of the SFS's at various EDF lengths when pumped at $80 \mathrm{~mW}$. No optical attenuation was applied in the measurement. It is noted that there was an optimal length of EDF for the maximum output power, 6- and 7-m long for DPF and DPB configurations, respectively. When the EDF 


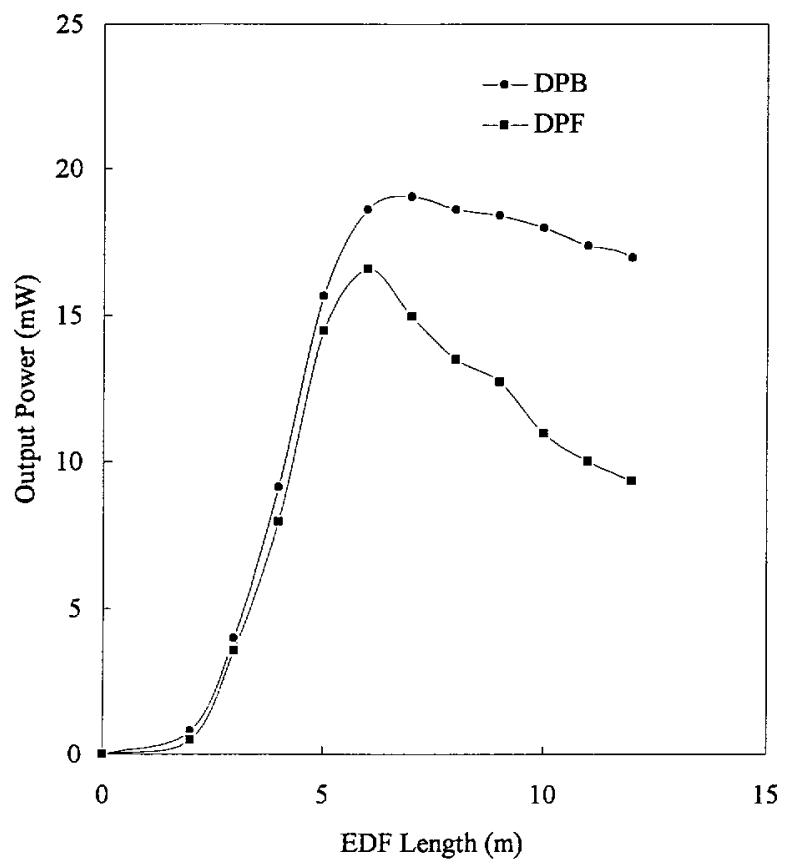

Fig. 2. Output power of double-pass SFS's at various EDF lengths.

was longer than the optimal length, the output power decayed owing to the reabsorption of ASE signals in the EDF. The output power in DPF configuration, however, decayed more rapidly than that in DPB one. As the EDF was long enough (e.g., $\geq \sim 18 \mathrm{~m}$ ), the output power of a DPB SFS eventually reached the same saturated level of an SPB SFS, independent of EDF length, because the effect of mirror reflectance became insignificant. In contrast, the output power of a DPF SFS continued decaying when the EDF length exceeded its optimal length. As also shown in Fig. 2, an SFS in DPB configuration would provide higher output power than that in DPF one at various EDF lengths.

The dependence of mean wavelength on pump power was characterized for SFS's in both configurations. The mean wavelength of a DPB SFS has been reported to have little or no pump-power dependence [6]. Quite different dependence was obtained from a DPF SFS at various EDF lengths as depicted in Fig. 3. The slopes in the high pump regions $(70-80 \mathrm{~mW})$ were about $-13,-13,-22,-32,-34$, and $-40 \mathrm{ppm} / \mathrm{mW}$ for $5,6,7,8,9$, and 10-m EDF's, respectively. Within the maximum pump power available, however, no $\partial \bar{\lambda}_{\text {source }} / \partial P_{\text {pump }}=$ 0 operation was found for the EDF lengths. The results therefore imply that to achieve the mean wavelength stability required by a navigation grade gyroscope, an SFS in DPF configuration may need to have a pump laser with much better power stability in addition to properly choosing the EDF length. It is noted that the linewidth decreased with the increasing pump power levels, and saturated at $\sim 12 \mathrm{~nm}$ in the high-pump regions for the optimal length of EDF $(6 \mathrm{~m})$.

The effects of fiber mirror reflectance on output power and linewidths for double-pass SFS's were characterized. The EDF lengths used in the DPB and DPF configurations were of 11 and $6 \mathrm{~m}$, respectively. The reasons for choosing such EDF lengths were because, at $80-\mathrm{mW}$ pump power,

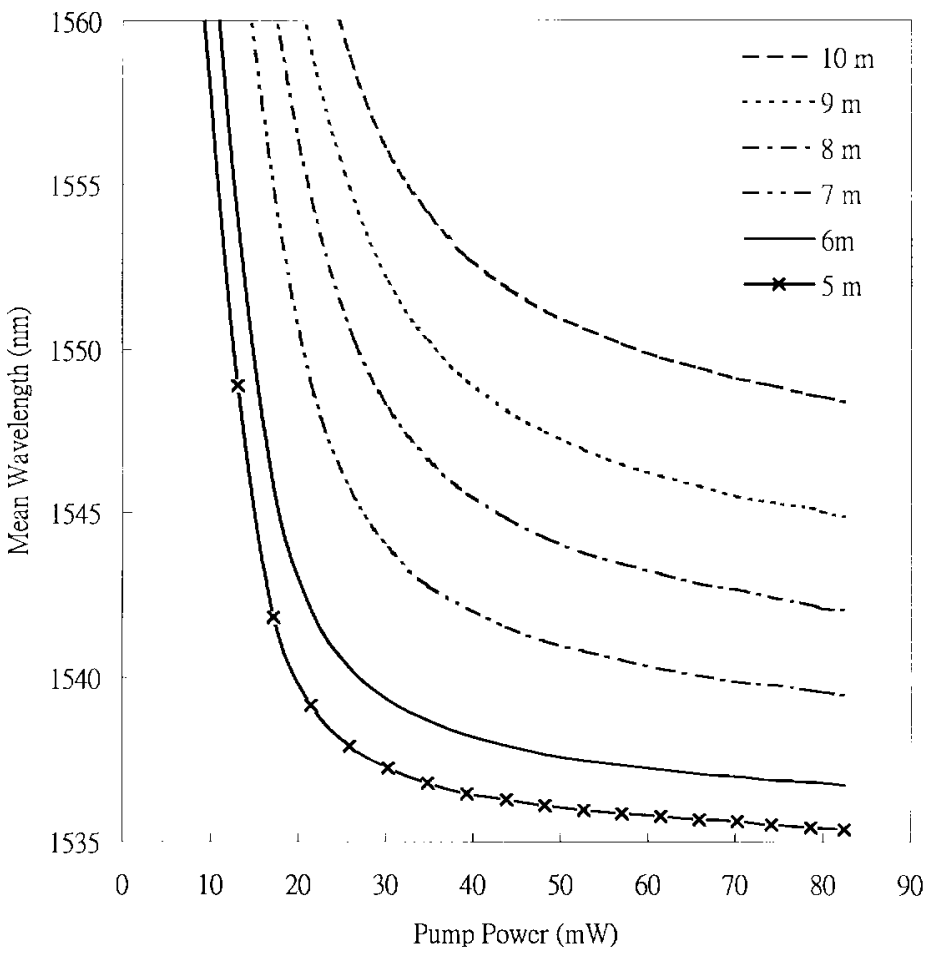

Fig. 3. Measured mean wavelength of a DPF SFS against pump power.

$\partial \bar{\lambda}_{\text {source }} / \partial P_{\text {pump }} \approx 0$ operation and high pump efficiency could be attained for the DPB and the DPF SFS's, respectively. The output power decreased as mirror reflectance became less in each configuration, not shown here, and eventually reached a floor where both double-pass configurations essentially behaved as single-pass ones. Fig. 4 shows the dependence of linewidth on the mirror reflectance. Note the reflectance here was corrected for the losses of the attenuator and the WDM. In DPF configuration, the linewidth decreased from $\sim 11$ $\mathrm{nm}$ to the minimum $(\sim 7 \mathrm{~nm})$ as the reflectance increased from -60 to $-30 \mathrm{~dB}$, and then increased to $\sim 12 \mathrm{~nm}$ for $\sim 50 \%$ reflectance. The spectra measured at various reflectance indicated that such effect was caused by the evolution of ASE signals around 1535 and $1550 \mathrm{~nm}$. The ASE signals around $1535 \mathrm{~nm}$ grew up faster than those around $1550 \mathrm{~nm}$ as the reflectance increased from -60 to $-30 \mathrm{~dB}$, leading to linewidth narrowing. The signals $\sim 1535 \mathrm{~nm}$ then became saturated as the reflectance increased toward $50 \%$, however, the signals $\sim 1550 \mathrm{~nm}$ kept increasing, thus broadening the linewidth. In contrast, the linewidth of the DPB SFS remained almost the same $(\sim 13 \mathrm{~nm})$ as the reflectance increased from -60 to $-25 \mathrm{~dB}$, and then increased to $27 \mathrm{~nm}$ as $50 \%$ mirror reflectance was reached. The rapid linewidth increase was caused by the drop of $\sim 1535 \mathrm{~nm}$ and the rise of $\sim 1550$ $\mathrm{nm}$ signals in such a way that both power levels became comparable. Since a high-power SFS is excess-noise limited [7], its signal-to-noise ratio (SNR) is solely determined by the source linewidth and the detector bandwidth. For navigationgrade FOG applications, a linewidth of $10 \mathrm{~nm}$ or an SNR equivalent of $\sim 128 \mathrm{~dB}$ for a detector with $500-\mathrm{kHz}$ bandwidth may be required for an Er-doped SFS. Therefore, as depicted in Fig. 4 an SFS in DPB rather than in DPF configuration is 




Fig. 4. Linewidths of DPB and DPF SFS's as a function of fiber mirror reflectance.

expected to achieve the required linewidth at various amounts of reflectance.

\section{CONCLUSION}

We have characterized and compared Er-doped SFS's pumped near $980 \mathrm{~nm}$ in double-pass forward and backward configurations. The results are summarized as the following. First, there is an optimal EDF length for the maximum output power in each configuration, and beyond that length the output power of a DPB SFS decays more slowly toward a constant level rather than continues decaying as in DPF one. In addition, for various lengths of EDF, an SFS in DPB configuration has higher output power. Second, a DPB SFS can have better pump-power mean wavelength stability while still maintaining a high-pump efficiency. Third, the various reflectance of fiber mirrors causes SFS's in both configurations to have different spectrum evolution, resulting in different linewidth variations. It is apparent that a DPB SFS has larger linewidths at various reflectance, all above the FOG's requirement. Therefore, a DPB SFS is expected to be a better choice when considering which double-pass configuration should be utilized as light source for navigation-grade gyroscopes.

\section{REFERENCES}

[1] P. F. Wysocki, M. J. F. Digonnet, B. Y. Kim, and H. J. Shaw, "Characteristics of erbium-doped superfluorescent fiber sources for interferometric sensor applications," J. Lightwave Technol., vol. 12, pp. 550-567, Mar. 1994.

[2] D. C. Hall and W. K. Burns, "Wavelength stability optimization in Erdoped superfluorescent fiber sources," Electron. Lett., vol. 30, no. 8, pp. 653-654, 1994.

[3] P. F. Wysocki, M. J. F. Digonnet, and B. Y. Kim, "Wavelength stability of a high-output, broadband, Er-doped superfluorescent fiber source pumped near 980 nm," Opt. Lett., vol. 16, no. 12, pp. 961-963, 1991.

[4] D. C. Hall, W. K. Burns, and R. P. Moeller, "High-stability Er-doped superfluorescent fiber sources," J. Lightwave Technol., vol. 13, pp. 1452-1460, July 1995.

[5] P. F. Wysocki, R. F. Kalman, M. J. F. Digonnet, and B. Y. Kim, "A comparison of 1.48 um and $980 \mathrm{~nm}$ pumping for Er-doped superfluorescent fiber sources," SPIE, vol. 1581, 1991, pp. 40-57.

[6] L. A. Wang and C. D. Chen, "Stable and broadband Er-doped superfluorescent fiber sources utilizing double-pass backward configuration," Electron. Lett., vol. 32, no. 19, pp. 1815-1817, 1996.

[7] P. R. Morkel, R. I. Laming, and D. N. Payne, "Noise characteristics of high-power doped-fiber superluminescent sources," Electron. Lett., vol. 26, no. 2, pp. 96-98, 1990. 